\title{
HUMAN AND PRE-HUMAN CULTURE AND THE EVOLUTION OF LANGUAGE
}

\author{
ANDREW WHITEN ${ }^{* 1}$ \\ *Corresponding Author: aw2@st-andrews.ac.uk \\ ${ }^{1}$ University of St Andrews, Fife, Scotland, UK
}

Human language is intimately linked with human culture. A child's language is culturally learned from others; and conversely, language can be a major vehicle of cultural transmission. To understand the evolution of language, we therefore need to understand the evolution of the capacity for culture. In this we are lucky, for by contrast with the apparent gulf between human language and all other animal communication, I shall describe how culture in some very recognisable forms has been discovered to be surprisingly widespread among animals and particularly rich in our closest primate relatives, from which we can make inferences about the ancestry of our cultural cognition. Tracing the evolution of culture is thus arguably a much more tractable empirical quest than that of language per se, and in this talk I will review research by my own and other research groups that pursues this through a multitude of different and convergent methods, from ethological observation in the wild to a diversity of cultural diffusion experiments, completed with both human and non-human primates in our own research. Mindful of the debate that pits a gestural against a vocal origin hypothesis for the evolution of language (the meeting of criteria for intentional communication argued to favour the gestural candidate), I note there is much evidence for vocal learning amongst varied animal taxa (although relatively little amongst primates) compared with little or none for the cultural transmission of gesture. This may suggest that vocal learning from others is 'easy' compared to copying gestural communication. Yet, perhaps paradoxically, the evidence for cultural transmission in primates is massively concerned with manual actions, such as those directed at functional, material outcomes, like tool use. I conclude that while they lack much sign of linguistictype communication, contemporary apes, and hence likely our early hominin 